\title{
Topological couplings in higher derivative extensions of supersymmetric three-form gauge theories
}

\author{
Muneto Nitta and Ryo Yokokura \\ Department of Physics 8 Research and Education Center for Natural Sciences, \\ Keio University, \\ Hiyoshi 4-1-1, Yokohama, Kanagawa 223-8521, Japan \\ E-mail: nitta@phys-h.keio.ac.jp, ryokokur@keio.jp
}

ABSTRACT: We consider a topological coupling between a pseudo-scalar field and a 3form gauge field in $\mathcal{N}=1$ supersymmetric higher derivative 3 -form gauge theories in four spacetime dimensions. We show that ghost/tachyon-free higher derivative Lagrangians with the topological coupling can generate various potentials for the pseudo-scalar field by solving the equation of motion for the 3-form gauge field. We give two examples of higher derivative Lagrangians and the corresponding potentials: one is a quartic order term of the field strength and the other is the term which can generate a cosine-type potential of the pseudo-scalar field.

KEywords: Superspaces, Supersymmetric Effective Theories

ARXiv EPrint: 1810.12678 


\section{Contents}

1 Introduction 1

2 3-form gauge theories with a topological coupling 3

2.13 -form gauge field 4

2.2 Topological coupling with a pseudo-scalar field 4

2.3 Higher derivative term and topological coupling 5

3 Higher derivative terms and scalar potentials in SUSY 3-form gauge theories

3.13 -form gauge field in superspace 8

$\begin{array}{llr}3.2 & \text { Scalar potential for a pseudo-scalar field } & 9\end{array}$

$\begin{array}{ll}3.3 & \text { Scalar potential from higher derivative terms } \\ & 11\end{array}$

$\begin{array}{lll}3.3 .1 & \text { General arguments } & 11\end{array}$

$\begin{array}{ll}\text { 3.3.2 Example 1: } \Lambda=\text { constant } & 14\end{array}$

$\begin{array}{lll}\text { 3.3.3 Example 2: cosine-type potential } & 16\end{array}$

$\begin{array}{lll}4 & \text { Summary } & 17\end{array}$

\section{Introduction}

3 -form gauge theories in four dimensions (4D) have been investigated in various contexts. In quantum chromodynamics (QCD), 3-form gauge theories have been applied to confinement [1, 2], an effective description of the Chern-Simons 3-form [2, 3], U(1) problem [4, 5], and strong $\mathrm{CP}$ problem $[6,7]$. In cosmology, the 3 -form gauge theories have been studied in the cosmological constant problem [8-13], quintessence [14, 15], and inflationary models [16-21]. The 3-form gauge theories are also related to condensed matter physics, e.g., superconductor [22] and quantum Hall effect [23].

A supersymmetric (SUSY) extension of the 3-form gauge theories was introduced in ref. [24], and then it was embedded into supergravity (SUGRA) [25-27] (see refs. [28-30] as a review). The SUSY 3-form gauge theories were also extensively studied with various applications: gaugino condensation in SUSY Yang-Mills theories [31-33], coupling with a membrane [34-36], alternative formulation of old-minimal SUGRA [34, 37-39], Stückelberg coupling [40-43], topological coupling [42, 44, 45], complex 3-form gauge theories [35, 36, 39], extended SUSY [46], the cosmological constant problem [38], SUSY breaking [38, 47, 48], and inflationary models [44, 49].

One of the virtues of the 3 -form gauge theories is that the 3 -form gauge field can generate a potential for a pseudo-scalar field with a topological coupling between the 3 -form and the pseudo-scalar field $[4,6,7,14,16,50]$. This mechanism was applied to effective 
theories of QCD [4, 6, 7, 51], cosmology [14-21, 52], and string effective theories [5355]. The pseudo-scalar field is often assumed to have a shift symmetry like a Goldstone boson or an axion, but a potential term generally break the shift symmetry of the pseudoscalar field. It was pointed out that the topological coupling preserves the shift symmetry of Lagrangians, and the shift symmetry is spontaneously broken $[14,16]$. A merit of this mechanism is that the potentials can be obtained by only assuming the symmetries of infrared theories, but not necessarily depend on details of ultraviolet models. This mechanism can also be considered in SUSY theories. In the SUSY theories, the field strength of the 3 -form gauge field can be embedded into an auxiliary field of a chiral superfield given by a real superfield [24]. The topological coupling is given by choosing appropriate superpotential [42, 44, 45]. This coupling was applied to SUGRA inflationary models [44, 49].

In the above applications, the 3-form gauge theories are often regarded as infrared (low energy or long range) effective theories. Since effective theories inevitably include nonrenormalizable interactions which generally depend on an ultraviolet (high energy or short range) cutoff parameter, it is natural to consider such nonrenormalizable terms in the 3 -form gauge theories. The nonrenormalizable terms may be given by functions of the field strength of the 3-form gauge field rather than the 3 -form gauge field itself because Lagrangians should be gauge invariant. Since the field strength is given by a spacetime derivative of the gauge field, the nonrenormalizable terms given by the field strength are regarded as higher derivative terms.

The higher derivative terms with the topological coupling in the 3-form gauge theories can generate various potentials for the pseudo-scalar field while preserving the shift symmetry for the pseudo-scalar field $[6,7,17,21]$. Indeed, a cosine-type potential for the pseudo-scalar field can be generated by choosing appropriate higher derivative terms [6]. This is in contrast to the case of the quadratic (canonical) kinetic term for which only the mass term (quadratic potential) for the pseudo-scalar field can be generated. This mechanism has been applied to the strong CP problem [6, 7] and inflationary models [17, 21]. For SUSY 3-form gauge theories, generating the potentials by the topological coupling and the higher derivative terms has been described in a quartic order of the field strength [44]. However, the general form of the potentials for the pseudo-scalar field has not been understood in our knowledge. The purpose of this paper is to investigate this general form of the potentials generated by the topological coupling and the higher derivative terms in SUSY 3-form gauge theories.

In the 3-form gauge theories, however, some higher derivative terms may lead to a tachyon instability when derivatives of the field strength are included in addition to the canonical kinetic term [56]. This instability is different from that of scalar field theories. For scalar field theories, derivative terms on a scalar field higher than the first order may give a so-called Ostrogradsky's ghost instability [57, 58]. While for the 3-form gauge theories, the derivative terms of the field strength of the 3-form gauge field [59-61] do not cause such ghosts in general [62] but may cause a tachyon. Note that the tachyon can also be a dynamical ghost depending on the parameter of the higher derivative term. This ghost is different from the Ostrogradsky's ghost. 
One of the sufficient conditions for the ghost/tachyon-free Lagrangians is that the Lagrangians should have an arbitrary function of the field strength but do not consist of derivative terms of the field strength of a 3-form gauge field [56]. Previously known models in refs. $[6,7,17,21]$ fall into this class. This condition is not a necessary condition: if the canonical kinetic term is absent or has the wrong sign [62], there is no tachyon instability. In SUSY 3-form gauge theories, the ghost/tachyon-free Lagrangians were considered in the quartic order of the field strength [44]. Later, the Lagrangians with an arbitrary order of the field strength were established [56]. Note that these SUSY Lagrangians are based on ghost-free Lagrangians for chiral superfields formulated in refs. [63-66], which have been applied in many contexts in SUSY effective field theories [67-91]. ${ }^{1}$

In this paper, we show a general framework and concrete examples of the generation of such the potentials by using the topological coupling and the higher derivative terms in bosonic and $\mathcal{N}=1$ SUSY 3-form gauge fields. For the bosonic 3-form gauge theories, we show a relation between the ghost/tachyon-free higher derivative terms and generated potentials. While the relation has been known by using dual formulations of the 3 -form gauge theories in ref. [6], we show a direct derivation which is given in terms of the 3 -form gauge field itself.

For the SUSY 3-form gauge theories, we use ghost/tachyon-free Lagrangians of the 3 -form gauge field with an arbitrary order of the field strength [56] in order to avoid the instabilities explained above. First, we give a bosonic part of the SUSY Lagrangian with the topological coupling and an arbitrary order of the field strength by using the ghost/tachyonfree Lagrangian of the 3-form gauge field. Second, we show a general expression of the potentials of the pseudo-scalar field generated by the topological coupling. Finally, we give two examples of the higher derivative terms and the generated potentials. One is a quartic order of the field strength. This quartic higher derivative term was described in ref. [44], and we derive the generated potential explicitly. Another is a cosine-type potential. We show that such the potential can also be obtained by choosing an appropriate SUSY higher derivative terms.

This paper is organized as follows. In section 2, we first review the potential generation mechanism in bosonic 3-form gauge theories. We then show a direct derivation of the generated potential [6] in terms of the 3-form gauge field itself. In section 3, we discuss the SUSY extension of the potential generation mechanism in the 3 -form gauge theories. In section 4, we summarize this paper. We use the notations and conventions of ref. [104].

\section{3-form gauge theories with a topological coupling}

In this section, we first review the role of the topological coupling with a pseudo-scalar field to generate a mass term (potential) of the pseudo-scalar field in 3-form gauge theories [4, $6,7,14,16]$. We then show the relation between the ghost/tachyon-free higher derivative terms and the generated potential in terms of the 3 -form gauge field itself rather than a

\footnotetext{
${ }^{1}$ For vector superfields, ghost-free higher derivative Lagrangians were considered in refs. [72, 92-97]. The ghost-free higher derivative Lagrangian with an arbitrary order of the field strength was formulated in ref. [98] and applied in refs. [99-103].
} 
dual formulation of the 3 -form gauge theories. A virtue of the 3 -form is a mass (potential) generation of a shift symmetric pseudo-scalar field.

\section{$2.1 \quad 3$-form gauge field}

A 3-form gauge field $C_{m n p}$ is a third-rank anti-symmetric tensor [1]. The gauge tranformation is given by

$$
\delta_{3} C_{m n p}=\partial_{m} \lambda_{n p}+\partial_{n} \lambda_{p m}+\partial_{p} \lambda_{m n},
$$

where $\delta_{3}$ denotes an infinitesimal gauge transformation of 3 -form gauge field, and $\lambda_{m n}$ is a second-rank anti-symmetric tensor parameter. To describe the kinetic term for the 3 -form gauge field, we introduce the field strength of the 3 -form gauge field as

$$
F_{m n p q}=\partial_{m} C_{n p q}-\partial_{n} C_{m p q}+\partial_{p} C_{m n q}-\partial_{q} C_{m n p}
$$

It is convenient to define the Hodge dual of the field strength $F:=\frac{1}{4 !} \epsilon^{m n p q} F_{m n p q}$, where $\epsilon^{m n p q}$ is the totally anti-symmetric tensor with the normalization $\epsilon^{0123}=+1$.

Then the quadratic (canonical) kinetic term for the 3-form gauge field is given by

$$
\mathcal{L}_{\text {kin. }}=-\frac{1}{2 \cdot 4 !} F^{m n p q} F_{m n p q}+\frac{1}{3 !} \partial_{m}\left(C^{m n p} F_{m n p q}\right)=+\frac{1}{2} F^{2}-\frac{1}{3 !} \partial_{m}\left(\epsilon_{m n p q} C^{m n p} F\right)
$$

with the following gauge invariant boundary condition for the 3 -form gauge field:

$$
\left.F\right|_{\text {bound. }}=-f_{0},
$$

where the symbol $\left.\right|_{\text {bound. }}$ denotes the value at the boundary, $f_{0}$ is a real constant, and the minus sign is just a convention. The term $\frac{1}{3 !} \partial_{m}\left(C^{m n p} F_{m n p q}\right)$ in eq. (2.3) is the boundary term for the 3 -form gauge field, which is needed for the vanishing of the functional variation of the 3-form gauge field at the boundary. If this boundary term were absent, the functional variation of the 3 -form gauge field would not vanish, and the equation of motion (EOM) for the 3 -form gauge field and the energy-momentum tensor would be inconsistent $[12,13]$.

\subsection{Topological coupling with a pseudo-scalar field}

In this subsection, we introduce a topological coupling between the 3-form gauge field and a pseudo-scalar field $[4,6,7,14,16]$. This coupling can be applied to the generation of a potential compatible with a shift symmetry for the pseudo-scalar field $[14,16]$. In general, the shift symmetry is broken by a potential term for the pseudo-scalar field. However, there is a mechanism of the generation of the potential which is compatible with the shift symmetry of the pseudo-scalar field if we consider a 3-form gauge field: the potential can be generated by a topological coupling between the 3 -form gauge field and the pseudo-scalar field $\phi$ of the form $\phi \epsilon^{m n p q} F_{m n p q}$.

The Lagrangian is given by

$$
\begin{aligned}
\mathcal{L}_{\text {top. }}= & -\frac{1}{2 \cdot 4 !} F^{m n p q} F_{m n p q}-\frac{1}{2} \partial^{m} \phi \partial_{m} \phi+\frac{1}{4 !} m \phi \epsilon^{m n p q} F_{m n p q} \\
& +\frac{1}{3 !} \partial^{m}\left(C^{n p q} F_{m n p q}-m \phi \epsilon_{m n p q} C^{n p q}\right)
\end{aligned}
$$


with the boundary condition for the pseudo-scalar field

$$
\left.\phi\right|_{\text {bound. }}=\phi_{0}
$$

and the one for the field strength in eq. (2.4). In eq. (2.5), $m$ is a mass-dimension one real parameter which will be a mass of the pseudo-scalar field $\phi$. The boundary term $+\frac{1}{3 !} \partial^{m}\left(C^{n p q} F_{m n p q}-m \phi \epsilon_{m n p q} C^{n p q}\right)$ is determined by the requirement that the variation of the 3 -form at the boundary should vanish.

The Lagrangian has a shift symmetry of the pseudo-scalar field

$$
\phi \rightarrow \phi+k,
$$

where $k$ is a real constant. Under the shift of the pseudo-scalar field, the Lagrangian is varied as

$$
\begin{aligned}
\mathcal{L}_{\text {top. }} & \rightarrow \mathcal{L}_{\text {top. }}+\frac{1}{4 !} m k \epsilon^{m n p q} F_{m n p q}-\frac{1}{3 !} m k \partial^{m}\left(\epsilon_{m n p q} C^{n p q}\right) \\
& =\mathcal{L}_{\text {top. }}+\frac{1}{4 !} m k \epsilon^{m n p q} F_{m n p q}-\frac{1}{4 !} m k \epsilon_{m n p q} F^{m n p q}=0 .
\end{aligned}
$$

Thus, the shift of the bulk term is canceled by that of the boundary term.

The generation of the mass term can be seen by solving EOM of the 3-form gauge field. The EOM of the 3-form gauge field is

$$
0=\partial^{m} F_{m n p q}-\epsilon_{m n p q} m \partial^{m} \phi
$$

Therefore, we can solve the EOM as

$$
F_{m n p q}=\epsilon_{m n p q}(m \phi+c),
$$

where $c$ is a constant determined by the boundary conditions for $F$ and $\phi$ as $c=f_{0}-m \phi_{0}$. Substituting this solution into the Lagrangian in eq. (2.5), we obtain

$$
\begin{aligned}
\mathcal{L}_{\text {top. }} & =+\frac{1}{2}(m \phi+c)^{2}-\frac{1}{2} \partial^{m} \phi \partial_{m} \phi-m \phi(m \phi+c)+\frac{1}{3 !} c \partial^{m}\left(\epsilon_{m n p q} C^{n p q}\right) \\
& =-\frac{1}{2} \partial^{m} \phi \partial_{m} \phi-\frac{1}{2}(m \phi+c)^{2}
\end{aligned}
$$

Therefore, the topological coupling gives us the mass of the pseudo-scalar field.

\subsection{Higher derivative term and topological coupling}

We have seen that the Lagrangian with the canonical kinetic term and the topological coupling can generate the mass term for the pseudo-scalar field. This mechanism can be generalized to more complicated potentials than the mass term. Such potentials can be generated by introducing higher derivative interactions for the 3 -form gauge field as shown below. We will consider a class of the higher derivative terms which do not cause either tachyon nor ghost instability. The higher derivative terms for the 3-form gauge fields often cause tachyon and/or ghost instability, although such the instability is absent if there are no derivative terms on the field strength. 
A ghost/tachyon-free higher derivative Lagrangian with the topological coupling can be written as

$$
\mathcal{L}_{\text {top.,HD }}=G(F)-\frac{1}{2} \partial^{m} \phi \partial_{m} \phi+m \phi F-\frac{1}{3 !} \epsilon^{m n p q} \partial_{m}\left(G^{\prime}(F) C_{n p q}+m \phi C_{n p q}\right) .
$$

Here, $G$ is an arbitrary function of the field strength [6, 7]. The canonical kinetic term can be included into $G$ as $G(F)=+\frac{1}{2} F^{2} \cdots$. The term $-\frac{1}{3 !} \epsilon^{m n p q} \partial_{m}\left(G^{\prime}(F) C_{n p q}+m \phi C_{n p q}\right)$ is the boundary term for the higher derivative term and the topological term. The boundary term for the higher derivative term has been determined by requiring the vanishing of the functional variation of the 3 -form at the boundary [56]. We assume the same boundary conditions for the field strength $F$ and the pseudo-scalar field $\phi$ as eq. (2.4) and eq. (2.6), respectively.

The potential for the pseudo-scalar field can be obtained by solving the EOM for the 3 -form gauge field as in the previous case. The EOM for the 3 -form gauge field is

$$
0=\partial_{m} G^{\prime}(F)+m \partial_{m} \phi
$$

This can be solved as

$$
-G^{\prime}(F)=m \phi+c,
$$

where $c$ is a constant determined by the boundary condition for the field strength and the pseudo-scalar field. By this equation, the field strength $F$ can be implicitly solved in terms of the pseudo-scalar field $\phi: F=F(\phi)$. Then, the Lagrangian becomes

$$
\mathcal{L}_{\text {top. }, \mathrm{HD}}=-\frac{1}{2} \partial^{m} \phi \partial_{m} \phi+G(F)-F G^{\prime}(F) .
$$

The term $G(F)-F G^{\prime}(F)$ is now the potential for the pseudo-scalar field. If we choose the function $G(F)$ as the canonical kinetic term as $G(F)=+\frac{1}{2} F^{2}$, we can obtain the mass term, since the solution to the EOM and the potential term $G(F)-F G^{\prime}(F)$ are $F+m \phi=-c$ and $-\frac{1}{2}(m \phi+c)^{2}$, respectively.

Generally, we can obtain an arbitrary potential for the pseudo-scalar field $V(\phi)$ by using this topological coupling [6]. The potential $V$ can be given in terms of the 3 -form gauge field as

$$
V(\phi)=-G(F)+F G^{\prime}(F) .
$$

The derivative of the both hand sides by $\phi$ is $V^{\prime}(\phi)=F G^{\prime \prime}(F) \frac{\partial F}{\partial \phi}$, and the derivative of eq. (2.14) by $\phi$ leads to

$$
V^{\prime}(\phi)=-m F
$$

Thus, the relation between the higher derivative term and the potential is

$$
V^{\prime}(\phi)=-m\left(G^{\prime}\right)^{-1}(-m \phi-c) .
$$

We should comment on the comparison with the previous work in ref. [6]. In the procedure in ref. [6], the dual description of the 3 -form was applied to derive eq. (2.18). Now, we have shown that the mechanism of the potential generation is directly obtained in terms of the 3 -form gauge field itself. 
As a concrete example, we review the generation of a cosine potential for the pseudoscalar field given in ref. [6]. The Lagrangian is given by

$$
\begin{aligned}
\mathcal{L}_{\mathrm{cos}}= & M^{2} F \arcsin \frac{F}{M^{2}}+M^{4} \sqrt{1-\frac{F^{2}}{M^{4}}}-\frac{1}{2} \partial^{m} \phi \partial_{m} \phi+m \phi F \\
& -\frac{1}{3 !} \epsilon^{m n p q} \partial_{m}\left(C_{n p q} M^{2} \arcsin \left(\frac{F}{M^{2}}\right)+m \phi C_{n p q}\right)
\end{aligned}
$$

where $M$ is a scale parameter with mass dimension one. Here, the higher derivative term has been chosen so that the relation between the potential and the higher derivative term in eq. (2.18) is satisfied:

$$
G(F)=M^{2} F \arcsin \frac{F}{M^{2}}+M^{4} \sqrt{1-\frac{F^{2}}{M^{4}}}
$$

The EOM for the 3-form gauge field is

$$
\frac{1}{\sqrt{1-\frac{F^{2}}{M^{4}}}} \partial_{m} F+m \partial_{m} \phi=0 .
$$

This can be solved as

$$
m \phi+c=-M^{2} \arcsin \left(\frac{F}{M^{2}}\right)
$$

where $c$ is a constant. Thus, we can express $F$ in terms of $\phi$ :

$$
F=-M^{2} \sin \left(\frac{m \phi+c}{M^{2}}\right)
$$

Substituting the solution into eq. (2.19), we obtain

$$
\mathcal{L}_{\cos }=-\frac{1}{2} \partial^{m} \phi \partial_{m} \phi+M^{4} \cos \left(\frac{m \phi+c}{M^{2}}\right) .
$$

\section{Higher derivative terms and scalar potentials in SUSY 3-form gauge theories}

In this section, we consider an $\mathcal{N}=1$ SUSY extension of the generation of the potentials of a pseudo-scalar field by the topological coupling and higher derivative terms for the 3 form gauge field. In this paper, we use superspace in order to formulate manifestly SUSY theories. The superspace is spanned by the bosonic spacetime coordinates $\left(x^{m}\right)$ and the fermionic coordinates given by Grassmann numbers $\left(\theta^{\alpha}, \bar{\theta}_{\dot{\alpha}}\right)$, where undotted and dotted Greek letters beginning with $\alpha, \beta, \ldots$ and $\dot{\alpha}, \dot{\beta}, \ldots$ denote undotted and dotted spinors, respectively. Thus, the coordinates of the superspace are denoted as $\left(x^{m}, \theta^{\alpha}, \bar{\theta}_{\dot{\alpha}}\right)$. We use the notation and convention of the ref. [104]. 


\subsection{3 -form gauge field in superspace}

Here, we briefly review SUSY 3-form gauge theories $[24,39,40]$. In the superspace, a 3-form gauge field $C_{m n p}$ is embedded into a real superfield $X$ satisfying $X^{\dagger}=X$ as follows:

$$
C_{m n p}=\frac{\sqrt{2}}{8} \epsilon_{m n p q}\left(\bar{\sigma}^{q}\right)^{\dot{\alpha} \alpha}\left[D_{\alpha}, \bar{D}_{\dot{\alpha}}\right] X \mid .
$$

Here, $\left(\bar{\sigma}^{q}\right)^{\dot{\alpha} \alpha}$ denotes $4 \mathrm{D}$ Pauli matrices, $D_{\alpha}$ and $\bar{D}_{\dot{\alpha}}$ are super-covariant spinor derivatives, and the vertical bar "|" denotes the $\theta=\bar{\theta}=0$ projection in the superspace. Hereafter, we will call $X$ "3-form prepotential" [28]. The gauge transformation of the 3 -form prepotential in the superspace is given by a chiral superfield parameter $\Upsilon_{\alpha}$ satisfying $\bar{D}_{\dot{\alpha}} \Upsilon_{\alpha}=0$ as

$$
\delta_{3, \mathrm{SUSY}} X=\frac{1}{2 i}\left(D^{\alpha} \Upsilon_{\alpha}-\bar{D}_{\dot{\alpha}} \bar{\Upsilon}^{\dot{\alpha}}\right)
$$

The field strength of the 3 -form gauge field is embedded into a chiral superfield $Y$, which is related to the original real superfield $X$ as follows:

$$
\begin{aligned}
Y & =-\frac{1}{4} \bar{D}^{2} X \\
F_{m n p q} & =\epsilon_{m n p q} \frac{\sqrt{2} i}{8}\left(D^{2} Y-\bar{D}^{2} \bar{Y}\right) \mid .
\end{aligned}
$$

Note that $Y$ is invariant under the gauge transformation in eq. (3.2). In the SUSY 3form gauge theories, there are superpartners of the 3-form gauge field, and they are also embedded into the chiral superfield $Y$. The superpartners are a complex scalar field $y=Y \mid$, a Weyl fermion $\chi_{\alpha}=\frac{1}{\sqrt{2}} D_{\alpha} Y \mid$, and a real auxiliary field $H=-\frac{\sqrt{2}}{8}\left(D^{2} Y+\bar{D}^{2} \bar{Y}\right) \mid$. It will be useful to define the combination of the field strength and the auxiliary field $H$ as

$$
\mathcal{F}=-\frac{1}{4} D^{2} Y \mid=\frac{1}{\sqrt{2}}(H-i F) .
$$

In SUSY 3-form gauge theories, we assume the boundary conditions for the 3-form prepotential. The boundary condition for the 3 -form gauge field is

$$
\left.\delta Y\right|_{\text {bound. }}=-\left.\frac{1}{4}\left(\bar{D}^{2} \delta X\right)\right|_{\text {bound. }}=0,
$$

where $\delta$ denotes the functional variation of fields. This boundary condition leads to $\left.\delta y\right|_{\text {bound. }}=\left.\delta \chi_{\alpha}\right|_{\text {bound. }}=\left.\delta H\right|_{\text {bound. }}=\left.\delta F\right|_{\text {bound. }}=0$. We further assume the same boundary condition for the field strength $F$ as

$$
\left.F\right|_{\text {bound. }}=-f_{0},
$$

which is consistent with the boundary condition in eq. (3.6).

The Lagrangian with the canonical kinetic term of the 3 -form gauge field can be written by

$$
\mathcal{L}_{\text {kin.,SUSY }}=-\frac{1}{8} \int d^{2} \theta \bar{D}^{2} Y \bar{Y}+\frac{i}{8}\left(\int d^{2} \theta \bar{D}^{2}-\int d^{2} \bar{\theta} D^{2}\right) T_{I} X+\text { h.c. }
$$


where $\int d^{2} \theta=-\frac{1}{4} D^{2} \mid$ is the so-called F-type integration. We use $-\frac{1}{8}\left(\int d^{2} \theta \bar{D}^{2}+\int d^{2} \bar{\theta} D^{2}\right)$ for the so-called D-type integration in stead of conventional $\int d^{4} \theta$ to fix the definition of the D-type integration. The first term leads to the canonical kinetic term of the 3 -form gauge field, while the second term gives us the boundary term for the first term. The superfield $T_{I}$ is the imaginary part of the chiral superfield $T: T_{I}=\frac{1}{2 i}(T-\bar{T})$, and $T$ is defined by

$$
T=-\frac{1}{4} \bar{D}^{2} \bar{Y}
$$

The chiral superfield $T$ is chosen by the requirement that the functional variation of the prepotential $X$ should vanish at the boundary.

The bosonic part of the Lagrangian can be expressed in terms of the component fields as follows:

$$
\mathcal{L}_{\text {kin.,SUSY }}=-\partial^{m} y \partial_{m} \bar{y}+\frac{1}{2} F^{2}+\frac{1}{2} H^{2}-\frac{1}{3 !} \partial_{m}\left(\epsilon^{m n p q} F C_{n p q}\right),
$$

where we have used the Wess-Zumino (WZ) gauge for the 3-form prepotential:

$$
X\left|=0, \quad D_{\alpha} X\right|=0, \quad \bar{D}_{\dot{\alpha}} X \mid=0 .
$$

Note that we hereafter omit fermions in the Lagrangians for the component fields, which will be irrelevant to the discussion of the generation of the potentials.

\subsection{Scalar potential for a pseudo-scalar field}

Here, we review the generation of the scalar potential for the pseudo-scalar field by topological coupling and the canonical kinetic term for the 3-form gauge field in SUSY field theories [44]. The pseudo-scalar field $\phi$ is embedded into a chiral superfield $\Phi$ :

$$
\phi=\frac{\sqrt{2}}{2 i}(\Phi-\bar{\Phi}) \mid
$$

The shift transformation of the pseudo-scalar field is given by

$$
\Phi \rightarrow \Phi+i k
$$

where $k$ is a real constant. In the SUSY theories, there are superpartners of the pseudoscalar field. The superpartners of the pseudo-scalar field are a real scalar field $s$, a Weyl fermion $\psi_{\alpha}$, and a complex auxiliary field $F_{\Phi}$. They are given by

$$
s=\frac{\sqrt{2}}{2}(\Phi+\bar{\Phi})\left|, \quad \psi_{\alpha}=\frac{1}{\sqrt{2}} D_{\alpha} \Phi\right|, \quad F_{\Phi}=-\frac{1}{4} D^{2} \Phi \mid .
$$

It will be convenient to define the following complex scalar field

$$
\varphi=\Phi \mid=\frac{1}{\sqrt{2}}(s+i \phi),
$$

which we will use in section 3.3. We assume the same boundary condition for the pseudoscalar field as eq. (2.6):

$$
\left.\phi\right|_{\text {bound. }}=\phi_{0} .
$$


Note that the boundary conditions for the other fields in $\Phi$ will not be used in the following discussion, therefore we do not show them explicitly.

The topological coupling between the pseudo-scalar field and the 3-form gauge field can be introduced by the superpotential $m \Phi Y$. The total Lagrangian is given by

$$
\begin{aligned}
\mathcal{L}_{\text {top. }, \text { SUSY }}= & -\frac{1}{8} \int d^{2} \theta \bar{D}^{2}\left(\frac{1}{2}(\Phi+\bar{\Phi})^{2}+Y \bar{Y}\right)+m \int d^{2} \theta \Phi Y \\
& +\frac{i}{8}\left(\int d^{2} \theta \bar{D}^{2}-\int d^{2} \bar{\theta} D^{2}\right) T_{I, \text { top. }} X+\text { h.c. }
\end{aligned}
$$

Here, the second line of the Lagrangian is the boundary term. The superfield $T_{I \text {,top. }}$ in the boundary term is the imaginary part of the chiral superfield $T_{\text {top. }}$ given by

$$
T_{\text {top. }}=-\frac{1}{4} \bar{D}^{2} \bar{Y}+m \Phi
$$

This chiral superfield is chosen so that the functional variation of the prepotential $X$ should vanish at the boundary. This Lagrangian is invariant under the shift transformation of the chiral superfield $\Phi$ in eq. (3.13). The shift transformations of the superpotential and the boundary term are canceled by each other, since these transformations are given by

$$
m \int d^{2} \theta \Phi Y+\text { h.c. } \rightarrow m \int d^{2} \theta \Phi Y+m i k \int d^{2} \theta Y+\text { h.c. }
$$

and

$$
\begin{aligned}
& \frac{i}{8}\left(\int d^{2} \theta \bar{D}^{2}-\int d^{2} \bar{\theta} D^{2}\right) T_{I, \text { top. }} X+\text { h.c. } \\
& \rightarrow \frac{i}{8}\left(\int d^{2} \theta \bar{D}^{2}-\int d^{2} \bar{\theta} D^{2}\right)\left(T_{I, \text { top. }}+m k\right) X+\text { h.c. } \\
& =\frac{i}{8}\left(\int d^{2} \theta \bar{D}^{2}-\int d^{2} \bar{\theta} D^{2}\right) T_{I, \text { top. }} X-\frac{i}{2} m k\left(\int d^{2} \theta Y-\int d^{2} \bar{\theta} \bar{Y}\right)+\text { h.c. }
\end{aligned}
$$

The scalar potential for the pseudo-scalar field can be seen by solving the EOM for the auxiliary fields and the field strength of the 3 -form gauge field. In order to obtain the EOM, it is convenitent to express the bosonic part of the Lagrangian. The bosonic part of the Lagrangian is

$$
\begin{aligned}
\mathcal{L}_{\text {top.,SUSY }}= & -\partial^{m} y \partial_{m} \bar{y}+\frac{1}{2} F^{2}+\frac{1}{2} H^{2}-\frac{1}{2} \partial^{m} \phi \partial_{m} \phi-\frac{1}{2} \partial^{m} s \partial_{m} s+F_{\Phi} \bar{F}_{\Phi} \\
& +m\left(y F_{\Phi}+\bar{y} \bar{F}_{\Phi}+F \phi+H s\right)-\frac{1}{3 !} \partial_{m}\left(\epsilon^{m n p q}(F+m \phi) C_{n p q}\right),
\end{aligned}
$$

where we have used the WZ gauge. The EOM for the field strength and the auxiliary fields are

$$
F+m \phi=-c, \quad H=-m s, \quad \bar{F}_{\Phi}=-m y .
$$

Substituting the solutions into the Lagrangian in eq. (3.21), we obtain

$$
\begin{aligned}
\mathcal{L}_{\text {top.,SUSY }}= & -\partial^{m} y \partial_{m} \bar{y}-\frac{1}{2} \partial^{m} \phi \partial_{m} \phi-\frac{1}{2} \partial^{m} s \partial_{m} s \\
& -m^{2}|y|^{2}-\frac{1}{2} m^{2} s^{2}-\frac{1}{2}(m \phi+c)^{2} .
\end{aligned}
$$


The last term is the mass term for the pseudo-scalar field. Note that the mass term for the scalar field $s$ is also generated due to SUSY.

\subsection{Scalar potential from higher derivative terms}

In this subsection, we consider the generation of the potentials for the pseudo-scalar field from the topological coupling and the ghost/tachyon-free higher derivative terms of the 3 -form gauge field, which is our new result in this paper.

In the previous sections 2.2 and 3.2, we have seen that the quadratic potential (mass term) can be generated by the quadratic (canonical) kinetic term of the 3 -form gauge field in the bosonic and SUSY 3-form gauge theories, respectively. Furthermore, we have reviewed in section 2.3 that more general potentials can be generated by higher derivative terms of the 3 -form gauge field. Thus, we can consider the potential generation mechanism in SUSY field theories.

Since a Kähler potential and a superpotential can only generate at most quadratic terms of the field strength, we should consider higher derivative terms of the 3 -form gauge field. We thus use the higher derivative term of the 3 -form gauge field proposed in ref. [56]. This higher derivative term gives us the terms of an arbitrary order of the field strength. Note that some higher derivative terms of the 3 -form gauge field such as $\partial^{m} F \partial_{m} F$ may cause tachyons and/or ghosts, although the higher derivative terms which we will use are free from such instabilities.

\subsubsection{General arguments}

Here, we consider a general expression of the scalar potential generated by the topological coupling and the higher derivative terms of the 3-form gauge field. A Lagrangian with the topological coupling and higher derivative terms is given by ${ }^{2}$

$$
\begin{aligned}
\mathcal{L}_{\mathrm{top}, \mathrm{HD}, \mathrm{SUSY}}= & -\frac{1}{8} \int d^{2} \theta \bar{D}^{2}\left(\frac{1}{2}(\Phi+\bar{\Phi})^{2}+Y \bar{Y}\right)+m \int d^{2} \theta \Phi Y \\
& -\frac{1}{8 \cdot 16} \int d^{2} \theta \bar{D}^{2} \Lambda\left(D^{\alpha} Y\right)\left(D_{\alpha} Y\right)\left(\bar{D}_{\dot{\alpha}} \bar{Y}\right)\left(\bar{D}^{\dot{\alpha}} \bar{Y}\right) \\
& +\frac{i}{8}\left(\int d^{2} \theta \bar{D}^{2}-\int d^{2} \bar{\theta} D^{2}\right) T_{I, \text { top.,HD }} X+\text { h.c. }
\end{aligned}
$$

Here, $\Lambda$ is a real function of $D^{2} Y$ and $\bar{D}^{2} \bar{Y}$ :

$$
\Lambda=\Lambda\left(D^{2} Y, \bar{D}^{2} \bar{Y}\right)
$$

The second line in eq. (3.24) is the higher derivative term for the 3-form gauge field. The last line in eq. (3.24) is the boundary term for the Lagrangian. The superfield $T_{I, \text { top.,HD }}$ in

\footnotetext{
${ }^{2}$ In this Lagrangian, we consider the simplest Kähler potential $\frac{1}{2}(\Phi+\bar{\Phi})^{2}+Y \bar{Y}$, although this quardratic kinetic term can be exnteneded to a general form $K(\Phi+\bar{\Phi}, Y, \bar{Y})$ while preserving the shift symmetry of the chiral superfield $\Phi$. The chiral superfield $\Phi$ can be dualized into a deformed real linear multiplet $L$ satisfying $L^{\dagger}=L$ and $-\frac{1}{4} \bar{D}^{2} L=m Y$ (see e.g. [44, 48]). The authors thank S. M. Kuzenko for comments on the generalization of the Kähler potential and the dual transformation.
} 
the last line is the imaginary part of the following chiral superfield

$$
\begin{aligned}
T_{\text {top.,HD }}= & -\frac{1}{4} \bar{D}^{2} \bar{Y}+m \Phi \\
& -\frac{1}{4 \cdot 16} \bar{D}^{2}\left(\frac{\partial \Lambda}{\partial Y}\left|D_{\alpha} Y\right|^{4}+D^{2}\left(\frac{\partial \Lambda}{\partial D^{2} Y}\left|D_{\alpha} Y\right|^{4}\right)\right. \\
& \left.\quad-2 D^{\alpha}\left(\Lambda\left(D_{\alpha} Y\right)\left(\bar{D}_{\dot{\alpha}} \bar{Y}\right)\left(\bar{D}^{\dot{\alpha}} \bar{Y}\right)\right)\right) .
\end{aligned}
$$

The superfield can be determined by requiring that the functional variation of the 3 -form prepotential at the boundary should vanish [56]. The bosonic part of the $\theta=\bar{\theta}=0$ component of the chiral superfield $T_{\text {top.,HD }}$ is

$$
\begin{aligned}
T_{\text {top.,HD }} \mid= & \overline{\mathcal{F}}+\frac{1}{\sqrt{2}} m(s+i \phi)+2 \Lambda|\mathcal{F}|^{2} \overline{\mathcal{F}}-2 \Lambda \overline{\mathcal{F}} \partial^{m} y \partial_{m} \bar{y} \\
& -4\left(\frac{\partial \Lambda}{\partial D^{2} Y} \mid\right)\left(|\mathcal{F}|^{4}-2 \partial^{m} y \partial_{m} \bar{y}|\mathcal{F}|^{2}+\left(\partial^{m} y \partial_{m} y\right)\left(\partial^{n} \bar{y} \partial_{n} \bar{y}\right)\right) .
\end{aligned}
$$

The Lagrangian in eq. (3.24) is invariant under the shift of the chiral superfield $\Phi \rightarrow \Phi+i k$, with a real constant $k$. This is because the higher derivative term only depends on $Y$, and does not break this shift symmetry of $\Phi$.

In order to discuss the potential for the pseudo-scalar field, we show the bosonic part of the Lagrangian in eq. (3.24):

$$
\begin{aligned}
\mathcal{L}_{\text {top.,HD }, \text { SUSY }}= & -\partial^{m} y \partial_{m} \bar{y}+\mathcal{F} \overline{\mathcal{F}}-\frac{1}{2} \partial^{m} \phi \partial_{m} \phi-\frac{1}{2} \partial^{m} s \partial_{m} s+F_{\Phi} \bar{F}_{\Phi} \\
& +m\left(y F_{\Phi}+\bar{y} \bar{F}_{\Phi}+\mathcal{F} \varphi+\overline{\mathcal{F}} \bar{\varphi}\right) \\
& +\Lambda\left(|\mathcal{F}|^{4}-2|\mathcal{F}|^{2} \partial^{n} y \partial_{n} \bar{y}+\left(\partial^{n} y \partial_{n} y\right)\left(\partial^{p} \bar{y} \partial_{p} \bar{y}\right)\right) \\
& -\frac{\sqrt{2}}{3 !} \partial_{m}\left(T_{I, \text { top. }, \mathrm{HD}} \mid \epsilon^{m n p q} C_{n p q}\right) .
\end{aligned}
$$

Here, we have used the complex auxiliary field $\mathcal{F}$, instead of $F$ and $H$, since it will be convenient to see the potentials for the pseudo-scalar field $\phi$ and its superpartner $s$.

Now, we see the mechanism of the potential generation in the higher derivative SUSY 3-form gauge theories. To obtain the potential, we solve the EOM for the field strength and auxiliary fields as in section 3.2. The solution to the EOM for the auxiliary field $F_{\Phi}$ is the same: $F_{\Phi}=-m \bar{y}$. While, the solutions to the EOM for $F$ and $H$ are deformed by the higher derivative terms as

$$
\begin{aligned}
-\frac{1}{\sqrt{2}} i c= & \overline{\mathcal{F}}+m \varphi+\frac{\partial \Lambda}{\partial \mathcal{F}}\left(|\mathcal{F}|^{4}-2|\mathcal{F}|^{2}\left(\partial^{m} y \partial_{m} \bar{y}\right)+\left(\partial^{m} y \partial_{m} y\right)\left(\partial^{n} \bar{y} \partial_{n} \bar{y}\right)\right) \\
& +2 \Lambda\left(|\mathcal{F}|^{2} \overline{\mathcal{F}}-\overline{\mathcal{F}} \partial^{m} y \partial_{m} \bar{y}\right)
\end{aligned}
$$

where $c$ is determined by the boundary conditions. Note that the EOM and thier solutions for $F$ and $H$ can be derived by the variation of the 3 -form pretpotential $X$ in the 
superspace [56]. The on-shell Lagrangian is therefore

$$
\begin{aligned}
\mathcal{L}_{\text {top.,HD }, \mathrm{SUSY}}= & -\partial^{m} y \partial_{m} \bar{y}+\mathcal{F} \overline{\mathcal{F}}-\frac{1}{2} \partial^{m} \phi \partial_{m} \phi-\frac{1}{2} \partial^{m} s \partial_{m} s-m^{2}|y|^{2} \\
& +\mathcal{F}\left(m \varphi+\frac{1}{\sqrt{2}} i c\right)+\overline{\mathcal{F}}\left(m \bar{\varphi}-\frac{1}{\sqrt{2}} i c\right) \\
& +\Lambda\left(|\mathcal{F}|^{4}-2|\mathcal{F}|^{2} \partial^{n} y \partial_{n} \bar{y}+\left(\partial^{n} y \partial_{n} y\right)\left(\partial^{p} \bar{y} \partial_{p} \bar{y}\right)\right)
\end{aligned}
$$

where we have implicitly substituted the solution in eq. (3.29) into $\mathcal{F}$ in the on-shell Lagrangian.

We consider the relation between the higher derivative terms of the 3 -form gauge field and the generated potentials for the pseudo-scalar field. The potential term can be simply seen by setting spacetime derivatives on the following fields to zero: $\partial_{m} y=\partial_{m} \bar{y}=$ $\partial_{m} s=\partial_{m} \phi=0$. When the derivatives on the fields are set to zero, the Lagrangian in eq. (3.28) becomes

$$
\begin{aligned}
\mathcal{L}_{\text {top.,HD,SUSY }}= & \mathcal{G}+F_{\Phi} \bar{F}_{\Phi}+m\left(y F_{\Phi}+\bar{y} \bar{F}_{\Phi}+\mathcal{F} \varphi+\overline{\mathcal{F}} \bar{\varphi}\right) \\
& -\frac{\sqrt{2}}{3 !} \partial_{m}\left(\epsilon^{m n p q} C_{n p q} \operatorname{Im}\left(\frac{\partial \mathcal{G}}{\partial \mathcal{F}}+m \varphi\right)\right) .
\end{aligned}
$$

Here, $\mathcal{G}=\mathcal{G}(\mathcal{F}, \overline{\mathcal{F}})$ is a real function of $\mathcal{F}$ and $\overline{\mathcal{F}}$ defined by

$$
\mathcal{G}=\mathcal{F} \overline{\mathcal{F}}+\Lambda|\mathcal{F}|^{4}
$$

which can be understood as a SUSY extension of $G(F)$ in eq. (2.12). The solution to the auxiliary field $\mathcal{F}$ is simplified as

$$
-\frac{1}{\sqrt{2}} i c=\overline{\mathcal{F}}+m \varphi+\frac{\partial \Lambda}{\partial \mathcal{F}}|\mathcal{F}|^{4}+2 \Lambda|\mathcal{F}|^{2} \overline{\mathcal{F}}
$$

This leads to the relation between $\mathcal{F}$ and the scalar field $\varphi$ as

$$
-\frac{1}{\sqrt{2}} i c-m \varphi=\frac{\partial \mathcal{G}}{\partial \mathcal{F}}
$$

Thus, the potential part of the on-shell Lagrangian is

$$
\mathcal{L}_{\text {top.,HD,SUSY }}=\mathcal{G}-\mathcal{F} \frac{\partial \mathcal{G}}{\partial \mathcal{F}}-\overline{\mathcal{F}} \frac{\partial \mathcal{G}}{\partial \overline{\mathcal{F}}}-m^{2}|y|^{2} .
$$

We thus obtain the relation between the on-shell potential of the scalar field $\mathcal{V}(\varphi, \bar{\varphi})$ and the function of the field strength $\mathcal{G}$ as

$$
\mathcal{V}=-\mathcal{G}+\mathcal{F} \frac{\partial \mathcal{G}}{\partial \mathcal{F}}+\overline{\mathcal{F}} \frac{\partial \mathcal{G}}{\partial \overline{\mathcal{F}}}
$$

which can be seen as a SUSY extension of the relation between the potential for the pseudoscalar field $V(\phi)$ and the function of the field strength $G(F)$ in eq. (2.16). Note that the relation between the auxiliary field $\mathcal{F}$ and the potential can be obtained from eq. (3.34) and the derivatives of the both sides of eq. (3.36) by $\varphi$ :

$$
\frac{\partial \mathcal{V}}{\partial \varphi}=-m \mathcal{F}
$$

which is a SUSY extension of eq. (2.17). 


\subsubsection{Example 1: $\Lambda=$ constant}

As a simple example, we consider the case where $\Lambda$ is a constant: $\Lambda=\Lambda_{0}$, which was described in ref. [44] and we will explicitly solve the EOM. In this choice of $\Lambda, \mathcal{G}$ is given by

$$
\mathcal{G}=|\mathcal{F}|^{2}+\Lambda_{0}|\mathcal{F}|^{4}
$$

while the on-shell potential $\mathcal{V}$ is

$$
\mathcal{V}=|\mathcal{F}|^{2}+3 \Lambda_{0}|\mathcal{F}|^{4}
$$

In order to obtain the potential, we express $\mathcal{F}$ in terms of $\varphi$ by solving the EOM. The EOM for $\mathcal{F}$ is

$$
-\frac{1}{\sqrt{2}} i c-m \varphi=\overline{\mathcal{F}}+2 \Lambda_{0}|\mathcal{F}|^{2} \overline{\mathcal{F}} .
$$

By using eq. (3.40), the auxiliary field $\mathcal{F}$ can be related to the Hermitian conjugate $\overline{\mathcal{F}}$ as

$$
\mathcal{F}=-\frac{\frac{1}{\sqrt{2}} i c+m \varphi+\overline{\mathcal{F}}}{2 \Lambda_{0} \overline{\mathcal{F}}^{2}}
$$

Substituting the Hermitian conjugate of the relation,

$$
\overline{\mathcal{F}}=-\frac{-\frac{1}{\sqrt{2}} i c+m \bar{\varphi}+\mathcal{F}}{2 \Lambda_{0} \mathcal{F}^{2}},
$$

into eq. (3.40), we obtain

$$
2 \Lambda_{0}\left(\frac{1}{\sqrt{2}} i c+m \varphi\right) \mathcal{F}^{3}+\left(-\frac{1}{\sqrt{2}} i c+m \bar{\varphi}\right) \mathcal{F}+\left(-\frac{1}{\sqrt{2}} i c+m \bar{\varphi}\right)^{2}=0 .
$$

This can be solved by using the Cardano's formula [74]

$$
\mathcal{F}=e^{-i \eta} \omega^{k} \sqrt[3]{-\frac{q}{2}+\sqrt{\left(\frac{q}{2}\right)^{2}+\left(\frac{p}{3}\right)^{3}}}-e^{-i \eta} \omega^{3-k} \sqrt[3]{\frac{q}{2}+\sqrt{\left(\frac{q}{2}\right)^{2}+\left(\frac{p}{3}\right)^{3}}},
$$

where

$$
m \varphi+\frac{i c}{\sqrt{2}}=r e^{i \eta}, \quad p=\frac{1}{2 \Lambda_{0}}, \quad q=\frac{r}{2 \Lambda_{0}}, \quad \omega^{3}=1, \quad k=0,1,2 .
$$

Here, $r>0$ and $\eta$ are real parameters. The on-shell potential for the scalar field $\mathcal{V}$ is the one obtained by substituting the solution in eq. (3.44) into the potential in eq. (3.39). Examples of the on-shell potentials are found in figures 1 and 2 for $k=0,1$, respectively (the potential for $k=2$ can be obtained by $c+m \phi \rightarrow-(c+m \phi)$ of the potential for $k=1$ ).

The higher derivative terms generally deform the vacuum structure. In particular, SUSY can be spontaneously broken in the vacuum by the effect of the higher derivative terms [96]. Here, we discuss the vacuum structure in this model. In the following discussion, we assume $\Lambda_{0}>0$ for the stability of the system.

As in ordinary chiral superfield cases, whether SUSY is preserved or broken can be examined by the condition whether $\mathcal{F}=0$ is consistent with the EOM of $\mathcal{F}$ or not. To 

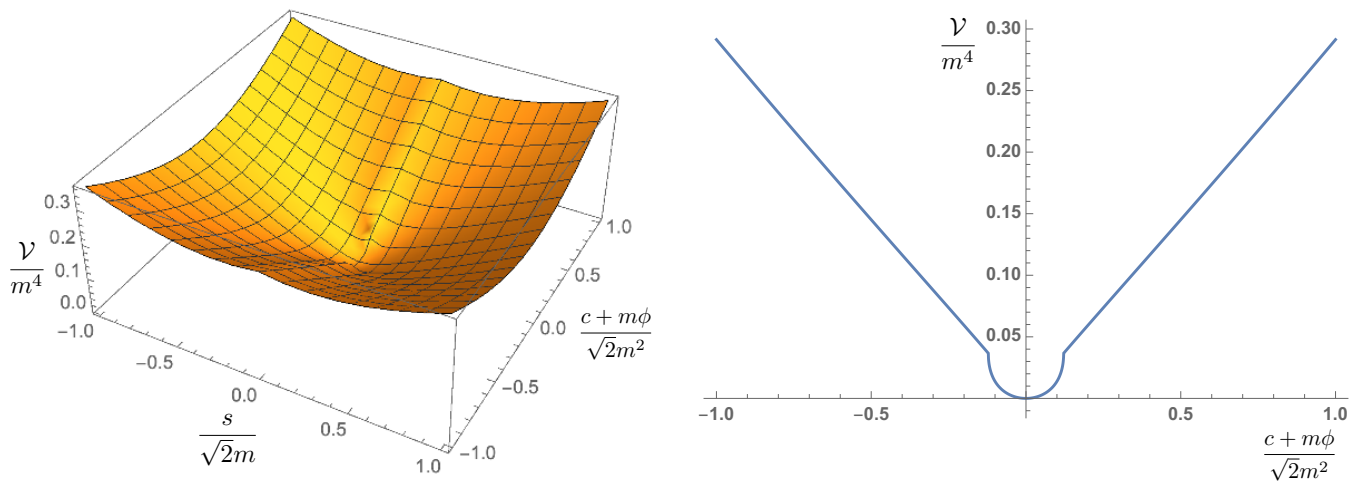

Figure 1. On-shell potential for $k=0$ with $m^{4} \Lambda_{0}=10$. The right figure corresponds to $s=0$ in the left figure.
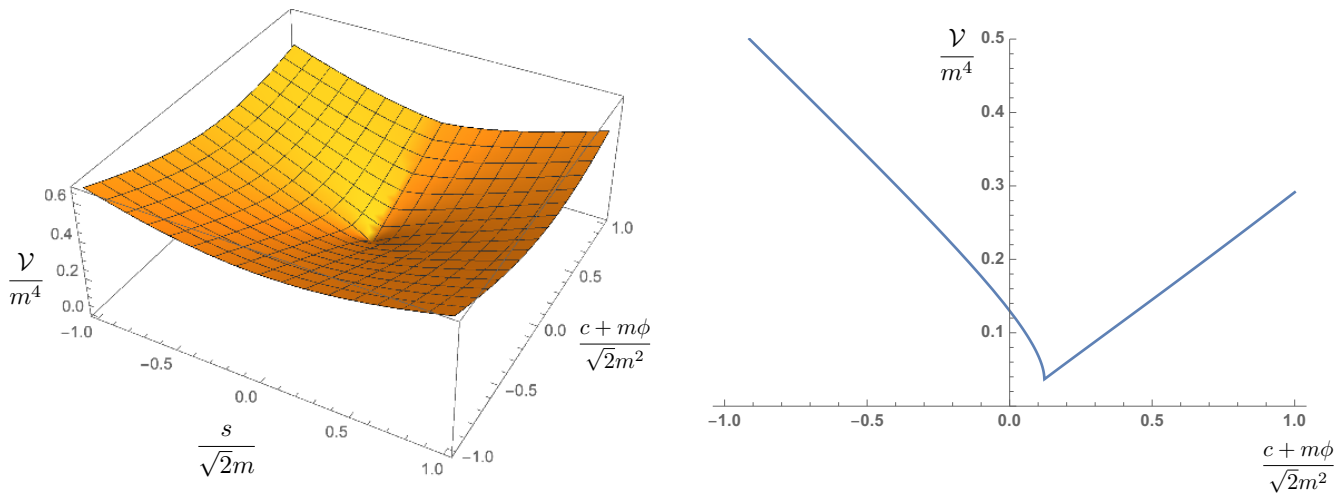

Figure 2. On-shell potential for $k=1$ with $m^{4} \Lambda_{0}=10$. The right figure corresponds to $s=0$ in the left figure.

examine it, let us assume $\mathcal{F}=0$. By the EOM for $\mathcal{F}$ in eq. (3.40), the assumption $\mathcal{F}=0$ leads to the condition for $\varphi$ :

$$
\varphi=-\frac{i c}{\sqrt{2} m}
$$

Substituting this condition into the EOM in eq. (3.44), one can show that the branch $k=0$ (called the canonical branch) is only consistent with the condition in eq. (3.46), while the branches $k=1,2$ (called the non-canonical branches) are not. This can be seen as follows. We express $\varphi$ around the condition in eq. (3.46) as $\varphi=-\frac{i c}{\sqrt{2} m}+\epsilon e^{i \eta_{0}}$, where $\epsilon$ is a real small parameter $\epsilon / m \ll 1$, and $\eta_{0}$ is an angle. The vacuum corresponds to $\epsilon=0$. The solution of the EOM in eq. (3.44) can be now expressed as

$$
\mathcal{F}=\omega^{k} e^{-i \eta_{0}} \frac{1}{\sqrt{6 \Lambda_{0}}}-\omega^{3-k} e^{-i \eta_{0}} \frac{1}{\sqrt{6 \Lambda_{0}}}+\mathcal{O}(\epsilon)
$$

and the branch $k=0$ only gives us the consistent solution $\mathcal{F}=0$. Therefore, the SUSY is preserved in the branch $k=0$, while SUSY is spontaneously broken in the branches $k=1,2$. Note that the values of $\mathcal{F}$ in the branches $k=1,2$ are $\mathcal{F}=e^{-i \eta_{0}} \frac{i}{\sqrt{2 \Lambda_{0}}},-e^{-i \eta_{0}} \frac{i}{\sqrt{2 \Lambda_{0}}}$, respec- 
tively, and the phase of $\mathcal{F}$ in the vacuum depends on the direction of a limit approaching to the vacuum in eq. (3.46).

\subsubsection{Example 2: cosine-type potential}

Next, we consider a cosine potential in SUSY 3-form gauge theories. In order to generate the cosine potential, we should choose a suitable real function $\Lambda$ so that the function $\mathcal{G}$ has the same structure as the function in eq. (2.20). We choose it as follows:

$$
\Lambda=\frac{1}{\left|-\frac{1}{4} D^{2} Y\right|^{4}}\left(M^{2} \hat{F} \arcsin \left(\frac{\hat{F}}{M^{2}}\right)+M^{4} \sqrt{1-\frac{\hat{F}^{2}}{M^{4}}}-\frac{1}{2} \hat{F}^{2}\right) .
$$

Here, $\hat{F}$ is defined by

$$
\hat{F}:=-\frac{\sqrt{2} i}{8}\left(D^{2} Y-\bar{D}^{2} \bar{Y}\right) .
$$

The $\theta=\bar{\theta}=0$ component of $\hat{F}$ is identified as the field strength $F$ :

$$
\hat{F}\left|=-\frac{\sqrt{2} i}{8}\left(D^{2} Y-\bar{D}^{2} \bar{Y}\right)\right|=F .
$$

In this choice, the function $\mathcal{G}$ is given by

$$
\mathcal{G}=M^{2} F \arcsin \left(\frac{F}{M^{2}}\right)+M^{4} \sqrt{1-\frac{F^{2}}{M^{4}}}+\frac{1}{2} H^{2},
$$

which is the same as the function in eq. (2.20). The on-shell potential in eq. (3.36) is therefore

$$
\mathcal{V}=-M^{4} \sqrt{1-\frac{F^{2}}{M^{4}}}+\frac{1}{2} H^{2}
$$

Note that we can choose the desired function $\mathcal{G}$ thanks to the denominator $\left|-\frac{1}{4} D^{2} Y\right|^{4}$ in $\Lambda$.

Now, we express the on-shell potential $\mathcal{V}$ in terms of $\varphi$ by solving the EOM for $\mathcal{F}$. The EOM for $\mathcal{F}$ is

$$
-\frac{1}{\sqrt{2}} i c-m \varphi=\frac{\partial \mathcal{G}}{\partial \mathcal{F}}=\frac{\partial H}{\partial \mathcal{F}} \frac{\partial \mathcal{G}}{\partial H}+\frac{\partial F}{\partial \mathcal{F}} \frac{\partial \mathcal{G}}{\partial F}=\frac{1}{\sqrt{2}} H-\frac{i}{\sqrt{2}} M^{2} \arcsin \left(\frac{F}{M^{2}}\right) .
$$

The real part of the equation gives us

$$
H=-m s,
$$

while the imaginary part gives us

$$
F=-M^{2} \sin \left(\frac{m \phi+c}{M^{2}}\right)
$$

Substituting the solutions into eq. (3.52), we obtain the Lagrangian with the following potential for $s$ and $\phi$ :

$$
\mathcal{V}=-M^{4} \cos \left(\frac{m \phi+c}{M^{2}}\right)+\frac{1}{2} m^{2} s^{2} .
$$

We thus have obtained the cosine potential for the pseudo-scalar field in addition to the mass term for $s$. 


\section{Summary}

In this paper, we have discussed a topological coupling of a 3-form gauge field with a pseudo-scalar field in SUSY higher derivative 3-form gauge theories. We have used a ghost/tachyon-free higher derivative Lagrangian of the 3-form gauge field. We have shown that the potential for the pseudo-scalar field is generated by substituting the solution of the EOM for the 3-form gauge field. We have presented two examples of the higher derivative Lagrangians and the corresponding potentials for the pseudo-scalar field. One is the quartic order of the field strength, which was described in ref. [44]. Another example is a SUSY extension of the bosonic model proposed in ref. [6], which generates a cosine potential for the pseudo-scalar field.

There can be some applications of the above Lagrangians. For example, we can apply them to SUGRA inflationary models. In this application, inflaton can be identified as the pseudo-scalar field. Since the pseudo-scalar field is shift-symmetric in the Lagrangian discussed in this paper, it can be a candidate for the origin for flatness of the inflaton potential. Furthermore, the higher derivative terms of the 3-form gauge field may play an important role to flatten the inflaton potential in SUGRA inflationary models as in the bosonic models in ref. [21].

In order to apply the mechanism of the generation of the potentials to the inflationary models, we should embed the topological coupling and the higher derivative terms into SUGRA. It will be convenient to use conformal SUGRA [105-111], since we can obtain the canonically normalized Einstein-Hilbert term by the superconformal gauge-fixing and avoid a tedious super-Weyl rescaling [107]. We will address the issue elsewhere.

In this paper, we have considered the potentials generated by the topological coupling and the higher derivative terms, but not considered the kinetic terms. In SUSY higher derivative theories of chiral superfields, it is known that kinetic terms in on-shell Lagrangians can also be deformed by higher derivative terms. If $\Lambda$ is a constant as in the example 1 in section 3.3, there are solutions to the auxiliary fields (so-called noncanonical branches) where the on-shell canonical kinetic term for the scalar field $y$ vanishes $[63,65,66,72,74,77]$ in addition to the conventional solution $\mathcal{F}=0$ (the canonical branch) giving rise to the usual kinetic term. It would be interesting to consider how the solutions are deformed in the presence of the topological coupling and the higher derivative terms with an arbitrary order of the field strength, which we have used in this paper.

\section{Acknowledgments}

This work is supported by the Ministry of Education, Culture, Sports, Science (MEXT)Supported Program for the Strategic Research Foundation at Private Universities "Topological Science" (Grant No. S1511006). M. N. is also supported in part by JSPS Grant-inAid for Scientific Research (KAKENHI Grant No. 16H03984 and No. 18H01217), and also by MEXT KAKENHI Grant-in-Aid for Scientific Research on Innovative Areas "Topological Materials Science" No. 15H05855. 
Open Access. This article is distributed under the terms of the Creative Commons Attribution License (CC-BY 4.0), which permits any use, distribution and reproduction in any medium, provided the original author(s) and source are credited.

\section{References}

[1] A. Aurilia and F. Legovini, Extended Systems and Generalized London Equations, Phys. Lett. 67B (1977) 299 [INSPIRE].

[2] A. Aurilia, The Problem of Confinement: From Two-dimensions to Four-dimensions, Phys. Lett. 81B (1979) 203 [INSPIRE].

[3] M. Lüscher, The Secret Long Range Force in Quantum Field Theories With Instantons, Phys. Lett. 78B (1978) 465 [INSPIRE].

[4] A. Aurilia, Y. Takahashi and P.K. Townsend, The U(1) Problem and the Higgs Mechanism in Two-dimensions and Four-dimensions, Phys. Lett. 95B (1980) 265 [INSPIRE].

[5] H. Hata, T. Kugo and N. Ohta, Skew Symmetric Tensor Gauge Field Theory Dynamically Realized in QCD U(1) Channel, Nucl. Phys. B 178 (1981) 527 [inSPIRE].

[6] G. Dvali, Three-form gauging of axion symmetries and gravity, hep-th/0507215 [INSPIRE].

[7] G. Dvali, A vacuum accumulation solution to the strong CP problem, Phys. Rev. D 74 (2006) 025019 [hep-th/0510053] [INSPIRE].

[8] A. Aurilia, H. Nicolai and P.K. Townsend, Hidden Constants: The Theta Parameter of QCD and the Cosmological Constant of $N=8$ Supergravity, Nucl. Phys. B 176 (1980) 509 [INSPIRE].

[9] S.W. Hawking, The Cosmological Constant Is Probably Zero, Phys. Lett. 134B (1984) 403 [INSPIRE].

[10] J.D. Brown and C. Teitelboim, Dynamical Neutralization of the Cosmological Constant, Phys. Lett. B 195 (1987) 177 [INSPIRE].

[11] J.D. Brown and C. Teitelboim, Neutralization of the Cosmological Constant by Membrane Creation, Nucl. Phys. B 297 (1988) 787 [InSPIRE].

[12] M.J. Duff, The Cosmological Constant Is Possibly Zero, but the Proof Is Probably Wrong, Phys. Lett. B 226 (1989) 36 [INSPIRE].

[13] M.J. Duncan and L.G. Jensen, Four Forms and the Vanishing of the Cosmological Constant, Nucl. Phys. B 336 (1990) 100 [inSPIRE].

[14] N. Kaloper and L. Sorbo, Where in the String Landscape is Quintessence, Phys. Rev. D 79 (2009) 043528 [arXiv:0810.5346] [INSPIRE].

[15] G. D'Amico, N. Kaloper and A. Lawrence, Strongly Coupled Quintessence, arXiv: 1809.05109 [INSPIRE].

[16] N. Kaloper and L. Sorbo, A Natural Framework for Chaotic Inflation, Phys. Rev. Lett. 102 (2009) 121301 [arXiv:0811.1989] [InSPIRE].

[17] N. Kaloper, A. Lawrence and L. Sorbo, An Ignoble Approach to Large Field Inflation, JCAP 03 (2011) 023 [arXiv:1101.0026] [InSPIRE].

[18] F. Marchesano, G. Shiu and A.M. Uranga, F-term Axion Monodromy Inflation, JHEP 09 (2014) 184 [arXiv: 1404.3040] [INSPIRE]. 
[19] N. Kaloper and A. Lawrence, Natural chaotic inflation and ultraviolet sensitivity, Phys. Rev. D 90 (2014) 023506 [arXiv: 1404.2912] [InSPIRE].

[20] N. Kaloper and A. Lawrence, London equation for monodromy inflation, Phys. Rev. D 95 (2017) 063526 [arXiv: 1607.06105] [INSPIRE].

[21] G. D'Amico, N. Kaloper and A. Lawrence, Monodromy Inflation in the Strong Coupling Regime of the Effective Field Theory, Phys. Rev. Lett. 121 (2018) 091301 [arXiv: 1709.07014] [INSPIRE].

[22] S. Ansoldi, A. Aurilia and E. Spallucci, Membrane vacuum as a type-II superconductor, Int. J. Mod. Phys. B 10 (1996) 1695 [hep-th/9511096] [inSPIRE].

[23] K. Hasebe, Higher Dimensional Quantum Hall Effect as A-Class Topological Insulator, Nucl. Phys. B 886 (2014) 952 [arXiv:1403.5066] [inSPIRE].

[24] S.J. Gates Jr., Super p-form gauge superfields, Nucl. Phys. B 184 (1981) 381 [INSPIRE].

[25] S.J. Gates Jr. and W. Siegel, Variant superfield representations, Nucl. Phys. B 187 (1981) 389 [INSPIRE].

[26] I.L. Buchbinder and S.M. Kuzenko, Quantization of the classically equivalent theories in the superspace of simple supergravity and quantum equivalence, Nucl. Phys. B 308 (1988) 162 [INSPIRE].

[27] P. Binetruy, F. Pillon, G. Girardi and R. Grimm, The three form multiplet in supergravity, Nucl. Phys. B 477 (1996) 175 [hep-th/9603181] [INSPIRE].

[28] S.J. Gates, M.T. Grisaru, M. Roček and W. Siegel, Superspace Or One Thousand and One Lessons in Supersymmetry, Front. Phys. 58 (1983) 1 [hep-th/0108200] [INSPIRE].

[29] I.L. Buchbinder and S.M. Kuzenko, Ideas and methods of supersymmetry and supergravity: Or a walk through superspace, IOP, Bristol, U.K., (1998).

[30] P. Binetruy, G. Girardi and R. Grimm, Supergravity couplings: A geometric formulation, Phys. Rept. 343 (2001) 255 [hep-th/0005225] [INSPIRE].

[31] P. Binetruy, M.K. Gaillard and T.R. Taylor, Dynamical supersymmetric breaking and the linear multiplet, Nucl. Phys. B 455 (1995) 97 [hep-th/9504143] [INSPIRE].

[32] P. Binetruy and M.K. Gaillard, $S$ duality constraints on effective potentials for gaugino condensation, Phys. Lett. B 365 (1996) 87 [hep-th/9506207] [INSPIRE].

[33] G.R. Dvali, G. Gabadadze and Z. Kakushadze, BPS domain walls in large N supersymmetric QCD, Nucl. Phys. B 562 (1999) 158 [hep-th/9901032] [INSPIRE].

[34] B.A. Ovrut and D. Waldram, Membranes and three form supergravity, Nucl. Phys. B 506 (1997) 236 [hep-th/9704045] [INSPIRE].

[35] S.M. Kuzenko and G. Tartaglino-Mazzucchelli, Complex three-form supergravity and membranes, JHEP 12 (2017) 005 [arXiv:1710.00535] [INSPIRE].

[36] I. Bandos, F. Farakos, S. Lanza, L. Martucci and D. Sorokin, Three-forms, dualities and membranes in four-dimensional supergravity, JHEP 07 (2018) 028 [arXiv:1803.01405] [INSPIRE].

[37] S.M. Kuzenko and S.A. McCarthy, On the component structure of $N=1$ supersymmetric nonlinear electrodynamics, JHEP 05 (2005) 012 [hep-th/0501172] [INSPIRE]. 
[38] F. Farakos, A. Kehagias, D. Racco and A. Riotto, Scanning of the Supersymmetry Breaking Scale and the Gravitino Mass in Supergravity, JHEP 06 (2016) 120 [arXiv:1605.07631] [INSPIRE].

[39] F. Farakos, S. Lanza, L. Martucci and D. Sorokin, Three-forms in Supergravity and Flux Compactifications, Eur. Phys. J. C 77 (2017) 602 [arXiv:1706.09422] [INSPIRE].

[40] K. Groh, J. Louis and J. Sommerfeld, Duality and Couplings of 3-Form-Multiplets in $N=1$ Supersymmetry, JHEP 05 (2013) 001 [arXiv:1212.4639] [INSPIRE].

[41] J. Hartong, M. Hubscher and T. Ortín, The supersymmetric tensor hierarchy of $N=1, d=4$ supergravity, JHEP 06 (2009) 090 [arXiv:0903.0509] [INSPIRE].

[42] K. Becker, M. Becker, W.D. Linch and D. Robbins, Abelian tensor hierarchy in $4 D, N=1$ superspace, JHEP 03 (2016) 052 [arXiv:1601.03066] [INSPIRE].

[43] S. Aoki, T. Higaki, Y. Yamada and R. Yokokura, Abelian tensor hierarchy in $4 D \mathcal{N}=1$ conformal supergravity, JHEP 09 (2016) 148 [arXiv: 1606.04448] [INSPIRE].

[44] E. Dudas, Three-form multiplet and Inflation, JHEP 12 (2014) 014 [arXiv:1407.5688] [INSPIRE].

[45] R. Yokokura, Abelian tensor hierarchy and Chern-Simons actions in $4 D \mathcal{N}=1$ conformal supergravity, JHEP 12 (2016) 092 [arXiv:1609.01111] [INSPIRE].

[46] N. Cribiori and S. Lanza, On the dynamical origin of parameters in $\mathcal{N}=2$ supersymmetry, Eur. Phys. J. C 79 (2019) 32 [arXiv:1810.11425] [INSPIRE].

[47] E.I. Buchbinder and S.M. Kuzenko, Three-form multiplet and supersymmetry breaking, JHEP 09 (2017) 089 [arXiv: 1705. 07700] [INSPIRE].

[48] S.M. Kuzenko, Nilpotent $\mathcal{N}=1$ tensor multiplet, JHEP 04 (2018) 131 [arXiv:1712.09258] [INSPIRE].

[49] Y. Yamada, U(1) symmetric $\alpha$-attractors, JHEP 04 (2018) 006 [arXiv: 1802.04848] [INSPIRE].

[50] T.L. Curtright and P.G.O. Freund, MASSIVE DUAL FIELDS, Nucl. Phys. B 172 (1980) 413 [INSPIRE].

[51] N. Kaloper and J. Terning, Landscaping the Strong CP Problem, JHEP 03 (2019) 032 [arXiv: 1710.01740] [INSPIRE].

[52] S. Franco, D. Galloni, A. Retolaza and A. Uranga, On axion monodromy inflation in warped throats, JHEP 02 (2015) 086 [arXiv:1405.7044] [INSPIRE].

[53] S. Bielleman, L.E. Ibáñez and I. Valenzuela, Minkowski 3-forms, Flux String Vacua, Axion Stability and Naturalness, JHEP 12 (2015) 119 [arXiv:1507.06793] [INSPIRE].

[54] I. Valenzuela, Backreaction Issues in Axion Monodromy and Minkowski 4-forms, JHEP 06 (2017) 098 [arXiv: 1611.00394] [INSPIRE].

[55] M. Montero, A.M. Uranga and I. Valenzuela, A Chern-Simons Pandemic, JHEP 07 (2017) 123 [arXiv: 1702.06147] [INSPIRE].

[56] M. Nitta and R. Yokokura, Higher derivative three-form gauge theories and their supersymmetric extension, JHEP 10 (2018) 146 [arXiv: 1809. 03957] [INSPIRE].

[57] M. Ostrogradsky, Mémoires sur les équations différentielles, relatives au problème des isopérimètres, Mem. Acad. St. Petersbourg 6 (1850) 385 [INSPIRE]. 
[58] R.P. Woodard, Avoiding dark energy with 1/r modifications of gravity, Lect. Notes Phys. 720 (2007) 403 [astro-ph/0601672] [INSPIRE].

[59] F.R. Klinkhamer and G.E. Volovik, Propagating q-field and q-ball solution, Mod. Phys. Lett. A 32 (2017) 1750103 [arXiv: 1609.03533] [inSPIRE].

[60] F.R. Klinkhamer and G.E. Volovik, Dark matter from dark energy in q-theory, JETP Lett. 105 (2017) 74 [arXiv:1612.02326] [INSPIRE].

[61] F.R. Klinkhamer and G.E. Volovik, More on cold dark matter from q-theory, arXiv: 1612.04235 [INSPIRE].

[62] F.R. Klinkhamer and T. Mistele, Classical stability of higher-derivative q-theory in the four-form-field-strength realization, Int. J. Mod. Phys. A 32 (2017) 1750090 [arXiv: 1704. 05436] [INSPIRE].

[63] J. Khoury, J.-L. Lehners and B. Ovrut, Supersymmetric $P(X, \phi)$ and the Ghost Condensate, Phys. Rev. D 83 (2011) 125031 [arXiv:1012.3748] [INSPIRE].

[64] J. Khoury, J.-L. Lehners and B.A. Ovrut, Supersymmetric Galileons, Phys. Rev. D 84 (2011) 043521 [arXiv:1103.0003] [INSPIRE].

[65] M. Koehn, J.-L. Lehners and B. Ovrut, Ghost condensate in $N=1$ supergravity, Phys. Rev. D 87 (2013) 065022 [arXiv: 1212.2185] [INSPIRE].

[66] M. Nitta and S. Sasaki, BPS States in Supersymmetric Chiral Models with Higher Derivative Terms, Phys. Rev. D 90 (2014) 105001 [arXiv:1406.7647] [InSPIRE].

[67] I.L. Buchbinder, S. Kuzenko and Z. Yarevskaya, Supersymmetric effective potential: Superfield approach, Nucl. Phys. B 411 (1994) 665 [InSPIRE].

[68] I.L. Buchbinder, S.M. Kuzenko and A.Yu. Petrov, Superfield chiral effective potential, Phys. Lett. B 321 (1994) 372 [INSPIRE].

[69] A.T. Banin, I.L. Buchbinder and N.G. Pletnev, On quantum properties of the four-dimensional generic chiral superfield model, Phys. Rev. D 74 (2006) 045010 [hep-th/0606242] [INSPIRE].

[70] S.M. Kuzenko and S.J. Tyler, The one-loop effective potential of the Wess-Zumino model revisited, JHEP 09 (2014) 135 [arXiv:1407.5270] [INSPIRE].

[71] M. Koehn, J.-L. Lehners and B.A. Ovrut, Higher-Derivative Chiral Superfield Actions Coupled to $N=1$ Supergravity, Phys. Rev. D 86 (2012) 085019 [arXiv:1207.3798] [INSPIRE].

[72] F. Farakos and A. Kehagias, Emerging Potentials in Higher-Derivative Gauged Chiral Models Coupled to $N=1$ Supergravity, JHEP 11 (2012) 077 [arXiv:1207.4767] [INSPIRE].

[73] J.M. Queiruga, Supersymmetric galileons and auxiliary fields in $2+1$ dimensions, Phys. Rev. D 95 (2017) 125001 [arXiv: 1612.04727] [InSPIRE].

[74] S. Sasaki, M. Yamaguchi and D. Yokoyama, Supersymmetric DBI inflation, Phys. Lett. B 718 (2012) 1 [arXiv: 1205.1353] [INSPIRE].

[75] S. Aoki and Y. Yamada, Inflation in supergravity without Kähler potential, Phys. Rev. D 90 (2014) 127701 [arXiv:1409.4183] [INSPIRE].

[76] S. Aoki and Y. Yamada, Impacts of supersymmetric higher derivative terms on inflation models in supergravity, JCAP 07 (2015) 020 [arXiv:1504.07023] [INSPIRE]. 
[77] C. Adam, J.M. Queiruga, J. Sanchez-Guillen and A. Wereszczynski, Extended Supersymmetry and BPS solutions in baby Skyrme models, JHEP 05 (2013) 108 [arXiv: 1304.0774] [INSPIRE].

[78] C. Adam, J.M. Queiruga, J. Sanchez-Guillen and A. Wereszczynski, $N=1$ supersymmetric extension of the baby Skyrme model, Phys. Rev. D 84 (2011) 025008 [arXiv:1105.1168] [INSPIRE].

[79] M. Nitta and S. Sasaki, Classifying BPS States in Supersymmetric Gauge Theories Coupled to Higher Derivative Chiral Models, Phys. Rev. D 91 (2015) 125025 [arXiv:1504.08123] [INSPIRE].

[80] S. Bolognesi and W. Zakrzewski, Baby Skyrme Model, Near-BPS Approximations and Supersymmetric Extensions, Phys. Rev. D 91 (2015) 045034 [arXiv:1407.3140] [INSPIRE].

[81] J.M. Queiruga, Baby Skyrme model and fermionic zero modes, Phys. Rev. D 94 (2016) 065022 [arXiv: 1606.02869] [INSPIRE].

[82] J.M. Queiruga, SUSY Chern-Simons $\mathbb{C P}^{N}$ and baby Skyrme models and their BPS structures, J. Phys. A 52 (2019) 055202 [arXiv:1807.09612] [INSPIRE].

[83] S.B. Gudnason, M. Nitta and S. Sasaki, A supersymmetric Skyrme model, JHEP 02 (2016) 074 [arXiv: 1512.07557] [INSPIRE].

[84] S.B. Gudnason, M. Nitta and S. Sasaki, Topological solitons in the supersymmetric Skyrme model, JHEP 01 (2017) 014 [arXiv:1608.03526] [INSPIRE].

[85] J.M. Queiruga, Skyrme-like models and supersymmetry in 3+1 dimensions, Phys. Rev. D 92 (2015) 105012 [arXiv: 1508.06692] [INSPIRE].

[86] J.M. Queiruga and A. Wereszczynski, Non-uniqueness of the supersymmetric extension of the $O(3) \sigma$-model, JHEP 11 (2017) 141 [arXiv: 1703.07343] [INSPIRE].

[87] M. Eto, T. Fujimori, M. Nitta, K. Ohashi and N. Sakai, Higher Derivative Corrections to Non-Abelian Vortex Effective Theory, Prog. Theor. Phys. 128 (2012) 67 [arXiv:1204.0773] [INSPIRE].

[88] M. Nitta and S. Sasaki, Higher Derivative Corrections to Manifestly Supersymmetric Nonlinear Realizations, Phys. Rev. D 90 (2014) 105002 [arXiv: 1408.4210] [INSPIRE].

[89] M. Nitta, S. Sasaki and R. Yokokura, Supersymmetry Breaking in Spatially Modulated Vacua, Phys. Rev. D 96 (2017) 105022 [arXiv:1706.05232] [INSPIRE].

[90] M. Nitta, S. Sasaki and R. Yokokura, Spatially Modulated Vacua in a Lorentz-invariant Scalar Field Theory, Eur. Phys. J. C 78 (2018) 754 [arXiv: 1706. 02938] [inSPIRE].

[91] F. Farakos, A. Kehagias and A. Riotto, Liberated $\mathcal{N}=1$ supergravity, JHEP 06 (2018) 011 [arXiv: 1805. 01877] [INSPIRE].

[92] S. Cecotti, S. Ferrara and L. Girardello, Structure of the Scalar Potential in General $N=1$ Higher Derivative Supergravity in Four-dimensions, Phys. Lett. B 187 (1987) 321 [INSPIRE].

[93] J. Bagger and A. Galperin, A new Goldstone multiplet for partially broken supersymmetry, Phys. Rev. D 55 (1997) 1091 [hep-th/9608177] [INSPIRE].

[94] S.M. Kuzenko and S. Theisen, Nonlinear selfduality and supersymmetry, Fortsch. Phys. 49 (2001) 273 [hep-th/0007231] [INSPIRE].

[95] S.M. Kuzenko and S.A. McCarthy, Nonlinear selfduality and supergravity, JHEP 02 (2003) 038 [hep-th/0212039] [INSPIRE]. 
[96] I. Antoniadis, E. Dudas and D.M. Ghilencea, Supersymmetric Models with Higher Dimensional Operators, JHEP 03 (2008) 045 [arXiv:0708.0383] [INSPIRE].

[97] E. Dudas and D.M. Ghilencea, Effective operators in SUSY, superfield constraints and searches for a UV completion, JHEP 06 (2015) 124 [arXiv: 1503.08319] [INSPIRE].

[98] T. Fujimori, M. Nitta, K. Ohashi, Y. Yamada and R. Yokokura, Ghost-free vector superfield actions in supersymmetric higher-derivative theories, JHEP 09 (2017) 143 [arXiv: 1708.05129] [INSPIRE].

[99] N. Cribiori, F. Farakos, M. Tournoy and A. van Proeyen, Fayet-Iliopoulos terms in supergravity without gauged R-symmetry, JHEP 04 (2018) 032 [arXiv:1712.08601] [INSPIRE].

[100] Y. Aldabergenov, S.V. Ketov and R. Knoops, General couplings of a vector multiplet in $N=1$ supergravity with new FI terms, Phys. Lett. B 785 (2018) 284 [arXiv:1806.04290] [INSPIRE].

[101] S.M. Kuzenko, Taking a vector supermultiplet apart: Alternative Fayet-Iliopoulos-type terms, Phys. Lett. B 781 (2018) 723 [arXiv:1801.04794] [INSPIRE].

[102] Y. Aldabergenov and S.V. Ketov, Removing instability of inflation in Polonyi-Starobinsky supergravity by adding FI term, Mod. Phys. Lett. A 91 (2018) 1850032 [arXiv:1711.06789] [INSPIRE].

[103] H. Abe, Y. Aldabergenov, S. Aoki and S.V. Ketov, Massive vector multiplet with Dirac-Born-Infeld and new Fayet-Iliopoulos terms in supergravity, JHEP 09 (2018) 094 [arXiv: 1808.00669] [INSPIRE].

[104] J. Wess and J. Bagger, Supersymmetry and supergravity, Princeton University Press, Princeton, U.S.A., (1992).

[105] E. Cremmer, S. Ferrara, L. Girardello and A. Van Proeyen, Yang-Mills Theories with Local Supersymmetry: Lagrangian, Transformation Laws and SuperHiggs Effect, Nucl. Phys. B 212 (1983) 413 [INSPIRE].

[106] T. Kugo and S. Uehara, Conformal and Poincaré Tensor Calculi in $N=1$ Supergravity, Nucl. Phys. B 226 (1983) 49 [inSPIRE].

[107] T. Kugo and S. Uehara, Improved Superconformal Gauge Conditions in the $N=1$ Supergravity Yang-Mills Matter System, Nucl. Phys. B 222 (1983) 125 [INSPIRE].

[108] T. Kugo and S. Uehara, $N=1$ Superconformal Tensor Calculus: Multiplets With External Lorentz Indices and Spinor Derivative Operators, Prog. Theor. Phys. 73 (1985) 235 [INSPIRE].

[109] D. Butter, $N=1$ Conformal Superspace in Four Dimensions, Annals Phys. 325 (2010) 1026 [arXiv:0906.4399] [INSPIRE].

[110] T. Kugo, R. Yokokura and K. Yoshioka, Component versus superspace approaches to $D=4$, $N=1$ conformal supergravity, PTEP 2016 (2016) 073B07 [arXiv: 1602.04441] [INSPIRE].

[111] T. Kugo, R. Yokokura and K. Yoshioka, Superspace gauge fixing in Yang-Mills matter-coupled conformal supergravity, PTEP 2016 (2016) 093B03 [arXiv:1606.06515] [INSPIRE]. 